\title{
Holistic approach of Brain-Mind interaction
}

\author{
Datta A K $\mathbf{K}^{1}$, Guha $\mathbf{R}^{2}$ \\ ${ }^{1}$ Emeritus Professor, ${ }^{2}$ Professor, Department of Anatomy, College of Medical Sciences, Bharatpur
}

\begin{abstract}
We live in three worlds - worlds 1, 2 and 3 with reciprocal interactions which make us perfect human beings.

World 1 is the word of physical objects; it comprises the whole of cosmos of matter and energy enriched with nature's contribution of flora and fauna, and all artefacts made by mankind in the form of books, machines, tools, arts and music.

World 2 deals with the states of consciousness and subjective perceptions at three levels of human brain in succession: (a) outer sense provided by all our sense organs that gives rise to the development of (b) inner sense in the form of our emotions, memories, imagination and planning for the future; (c) finally at the core of world 2 there develops a sense of consciousness for self or ego.

World 3 is created by man with the development of language of communication that uniquely relates to man. It is the world which is completely unknown to animals. All our means of communication and intellectual efforts are coded in books, the artistic and technological treasures are stored in the museum, and every artifact coded by man is preserved in the world 3. It is the world of civilization and culture. Education is the means whereby each human being is immersed in the world 3 throughout life, participating in the heritage of mankind and so becoming fully human.
\end{abstract}

Correspondence: Datta A K

Email: 


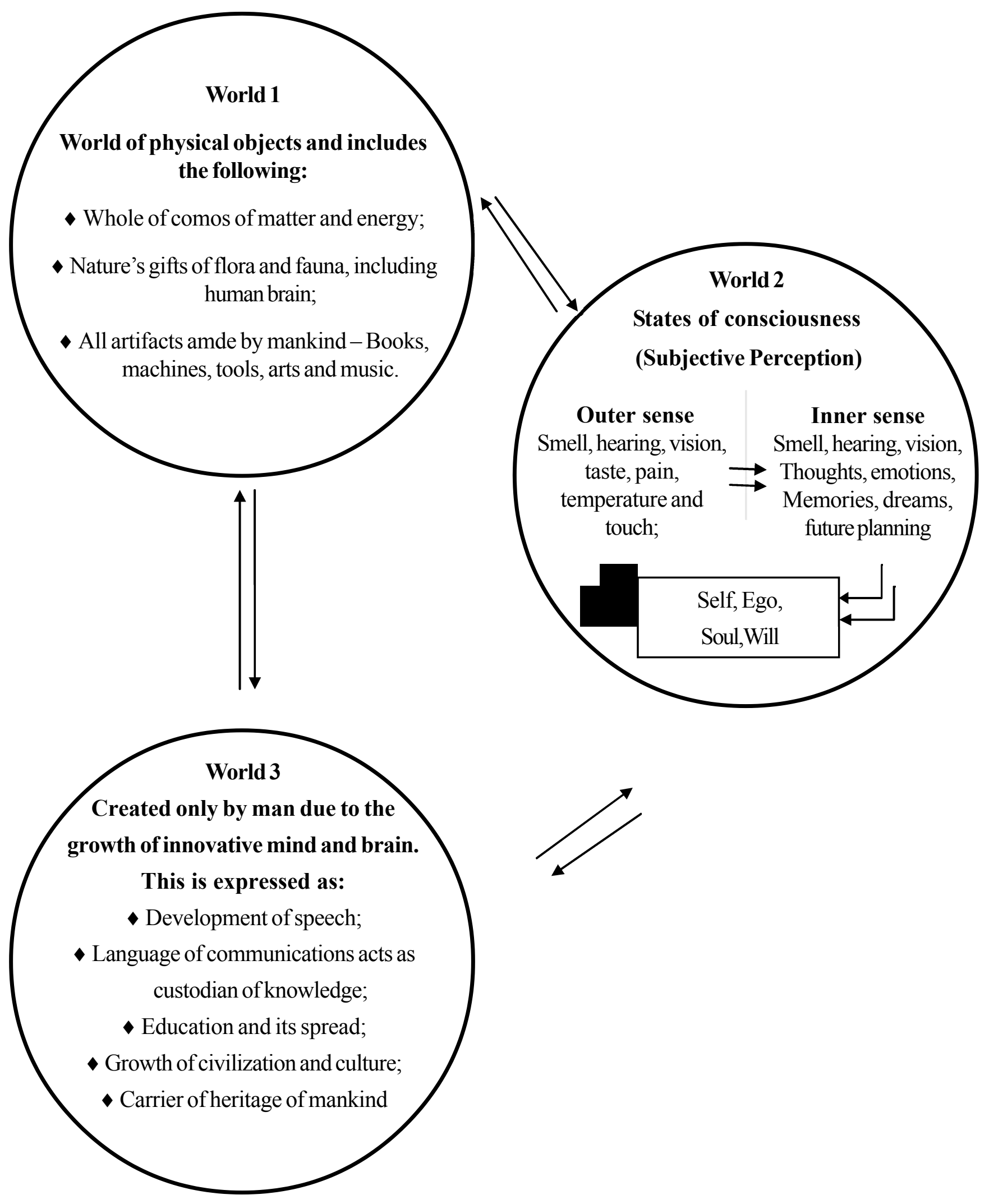

Holistic approach of Brain - Mind interaction 
Datta A K et al. Holistic approach of Brain..........

\section{Introduction}

When we, as intelligent human beings of the modern era, started thinking deeply and consciously about selfanalysis, we turned to be philosophers and wanted to establish a relationship and interaction between the brain and mind within ourselves. This philosophical problem leads to the development of the concept that we are living in three worlds - worlds 1,2 and 3. Thanks to the postulation of such concept by Sir Karl Popper1 which was fully accepted by the Novel Laureate. Sir John C. Eccles 2 and other distinguist bio-scientists.

World 1 comprises the whole cosmos of matter and energy, all of biology including human brains, and all artefacts that man has made for coding information in the form of books, machines, tools, art and music. Therefore, world 1 is the world of physical objects and states, and composed totally of materialists. ${ }^{1,2}$

World 2 is the world of states of consciousness and subjective knowledge of all kinds. Our perceptions comes in this world but they appear at several levels in succession. ${ }^{3,4}$

- The first level is the outer sense which consists of ordinary perceptions provided by all our sense organs in the form of smell, hearing, sight, pain, touch and so on.

- Next is the level of inner sense. It is the world of our emotions with feelings of joy and sadness, all kinds of memory and all our imaginations and planning for the future. This can be revealed in linguistic expression, and by gestures of all levels of subtlety.

- Finally at the core of world 2 there develops sense of self or ego which will persist throughout our whole life time.

World 2 is our primary reality, and the basis of knowledge with conscious experiences derived from world 1, which thus acts as a world of secondary reality. We are all the same, in every action we do, incessantly moving backwards and forwards between world 1 and world 2. ${ }^{1-3}$

World 3 is the whole world of culture. It is the world that was created by man and that reciprocally made man. The whole of language develops here. All our means of communication and intellectual efforts are coded in books, the artistic and technological treasures and stored in the museum, and every artefact coded by man are preserved in world 3. It is the world of civilization and culture. Education is the means whereby each human being is immersed in world 3 throughout life, participating in the heritage of mankind and so becoming fully human. World 3 is the world that uniquely relates to man. It is world which is completely unknown to animals. ${ }^{1,3}$

\section{Interaction of worlds 1, 2 and 3}

In each of us there is a continuous and intense interaction and flow of information between the three worlds: worlds $1 \rightleftarrows$ world 2; and $\rightleftarrows$ world $3 \rightleftarrows$ world $1 \rightleftarrows$ world 2. Transmission from world 1 to world 2 occurs through processes of sensing, coded transmission to the brain and decoding there.

Central theme of the brain - mind interaction would be the genesis of ego or self, which gives each of us unity as a person and religious sense may be expressed as soul. In cognitive experience there is outer sensing or perception via the receptor organs, inner sensing which is consolidated as memories in conscious states, and finally integration of all neuronal activities which make each of us to realise as a person or self. ${ }^{2,4}$ 
Journal of College of Medical Sciences-Nepal, 2014, Vol-10, No-1

Sperry ${ }^{5,6}$ and his associates made surgical section of the corpus callosum in human brains in patients suffering from severe epilepsy in order to confine the epileptic seizures to one hemisphere. This operation was performed in more than 20 human patients with admirable success. The patients did not suffer from changes in intellect, behaviour or emotional disturbances after commissurectomy. Transfer of learning processes and speech function is, however, much affected.

Sperry ${ }^{5,6}$ made a critical analysis in split - brain or twin brain patients after commissurectomy and postulated distinctive functions of the left and right hemispheres.

Left cerebral hemisphere receives input from and provides output to the right side of the body, and reverse is the case in the right hemisphere. When the right hand is doing some precision work, the left hand should have some knowledge about the activities of right hand through the inter-hemispheric commissure. Since more than $90 \%$ of human population uses the right hand in preference to the left hand for skilful works, the left hemisphere is considered to be dominant and eventually the right hemisphere serves as the non-dominant (minor) part of the brain.

The Dominant hemisphere (left) is considered with consciousness for self, linguistic expression (talking brain) and analysis of diverse information. It also solves arithmetical and algebric problems, and carries out other computer- like operations. The dominant hemisphere is associated with the existence of the ego or self with all memories of the past and acts as brain mind liaison. ${ }^{2,3}$

The dominant hemisphere presents four speech centres which are connected to one another and to the thalamus and corresponding areas of the right hemisphere. The centres are as follows. ${ }^{7,8}$

1. Area 22 in the posterior part of the superior temporal gyrus (wernicke's area) comprehends spoken language and recognizes familiar sounds and words.

2. Area 39 of the angular gyrus stores visual images and recognizes the objects by vision.

3. Area 40 of the supra-marginal gyrus recognizes familiar objects with the help of touch and proprioception.

The aforesaid three areas $(22,39,40)$ are in structural continuity and act as sensory speech area which receives input from hearing, vision, touch and proprioceptions. These information are further processed in the area 22, and thereafter projected to teh Broca's area through the arcuate fasciculus (gaschwind's theory). ${ }^{9}$

4) Areas 44 and 45 of inferior frontal gyrus of the dominant hemisphere (Broca's area) act as motor speech centre which lies immediately rostral to the motor cortex for the tongue, lips, larynx and pharynx. After receiving the input from the sensory speech centres the broca's area through facial region of the motor cortex and corticobulbar tract, stimulates the lower motor neurons of the brain stem for the co-ordinated movements of the spoken speech. When one speaks, the whole brain speaks. Speech function is mostly controlled by the dominant or left hemisphere.

Because of the partial decussation in the optic chiasma, the right visual field for both eyes projects to the visual cortex. In split - brain patients after commissurectomy, 
Datta A K et al. Holistic approach of Brain.......... sperry tested the level of visual consciousness of the patient by adopting the following method:

The subject in sitting position fixes his gaze on a central spot on the screen and on the left or right side of this screen some signal can be flashed for one - tenth of second. Now a message (say word) HAT BAND is projected on the screen so that HAT lies in the left visual field and BAND in the right visual field; thus HAT goes to the right hemisphere and BAND to the left (speaking) hemisphere. When you ask the subject what word did he see, he would say "band". This method of testing shows that the dominant left hemisphere is conscious for "self".

The non- dominant (right) hemisphere function in all kinds of geometrical and spatial arrangements in three dimensional perspectives. Moreover it is concerned with musical sense, artistry and synthesis of coherent thoughts.

Split brain patients cannot understand the movements performed by their two hands, after closure of both eyes. If you ask the patient to un-button his coat, by one hand he starts to unbutton while his other hand attempts to button it. Therefore he is unable to open the coat.

We have mentioned in the earlier part of this topic that world 3 is uniquely human. We should not accept the idea that man is just superior animal. It is true that man has outgrown his animal ancestry in intellectual, rational, ethical and aesthetic activities to an amazing degree. But to develop the uniqueness of man in world 3 from poor hominid state requires a million years to pass.

Man had developed considerable linguistic means of communication more than 100 millennia ago. Eventually he achieved self consciousness by this communication with his fellows. The primitive man would be looking at others dying and thinking and developed fellow feelings by this method. these linguistic usages might be creative kinds of language, not just "ooh" and "ah" with calling and warning cries, but a meaningful language .A developing language would give selective advantage to primitive men, first in there clubbing together in tribes and second in their communal grouping for hunting and fighting .progressive cerebral development along with linguistic expression by cross communication helps constructions of tools used for skilled control of movements. The consequent development of technology marks the origin of civilization. The creative imagination ofman is expressed in literature, art, architecture, and in the further developments in religion, philosophy and science. ${ }^{1,2}$

\section{CONCLUSION}

Gradual information collected centuries after centuries from world 1 makes man conscious about self. The progressive growth of brain with prolific potentialities to learn various aspects from the reciprocal interaction between world 2 and world 3 establishes him to become a cultured and civilized human being. This imposes a greater responsibility in his mind to preserve and enrich all the elements of physical objects and states in world 1 in which he grows and develops.

\section{REFERENCES}

1. Popper KR, and Eccles JC. The Self and its Brain ;1976 : Springer-Verlag, Heidelberg, London.

2. Eccles JC. The understanding of the Brain; $2^{\text {nd }}$ ed, Mc Graw-Hill Book Company 1977;192-228. 
Journal of College of Medical Sciences-Nepal, 2014, Vol-10, No-1

3. Eccles JC. The Neurophysiologist Basis of Mind : In the principles of Neurophysiology; 1953 : clarendon Press, Oxford.

4. Eccles JC. In Brain and conscious Experience; 1966 : Springer, Heidelberg, London.

5. Sperry RW. A Modified concept of consciousness; Psychol. Rev., 1969;76 : 532 - 6.

6. Sperry RW. "Lateral Specialization in the Surgically Separated Hemispheres"; The Neurosciences : Third study Program, : MIT Press, Cambridge, 1974; 5-19.
7. Penfield W, Roberts L. Are Speech and Brain Mechanisms; 1959: Princeton University Press, Princeton.

8. Geschwind N. "Language and the Brain", Sci, Amer, 1972; 226 :76-83

9. Geschwind N. "The Anatomical Basis of hemispheric Differentiation", in Diamond SJ. And Beaumont JG. (eds.), Hemisphere Function in the Human Brain; Wiley, New York, 1974;7-24.

10. Gazzaniga MS. "The Split Brain in Man", Sci. Amer: reprinted in Physiological Psychology. 1971;118-28. 